\title{
Correspondence
}

\section{Factitious readings from anaesthetic agent monitors}

To the Editor:

The use of anaesthetic agent monitoring as a component of airway gas monitoring has recently become commonplace in some hospitals. In principle, anaesthetic agent monitoring may alert the anaesthetist to possible differences between intended and obtained anaesthetic concentrations, as well as provide information regarding anaesthetic agent uptake and release.

I would like to report two cases of factitious readings from such a monitor. In the first case, shortly after intubation and hook-up to a Puritan Bennett PB254 Airway Gas Monitor (set for isoflurane mode) readings of approximately one per cent isoflurane were obtained for both inspiratory and expiratory readings, despite the fact that the vaporizer had not yet been turned on. In the second case, no anaesthetic agent had been selected, as the monitor was used to assess respiration in a heavily sedated patient whose procedure was being done under local anaesthesia. In this case airway gas sampling was achieved using a nasal catheter. Shortly after the monitor was connected, the front panel anaesthetic agent selection lights flashed and an audio alarm sounded, indicating that the monitor sensed the presence of an anaesthetic agent and was requesting that the operator select the agent used. To silence the alarm it was necessary to select an agent; halothane was chosen arbitrarily, even though no vaporizers were on. A reading of approximately $6 \%$ halothane was then obtained for both inspiratory and expiratory readings.

In both cases the anaesthetic machines had been checked preoperatively and no fault was found. As well, when the unexpected anaesthetic concentration readings were obtained, no evidence of malfunctioning vaporizers could be found.

However, in both cases when the anaesthetic circuits were opened and smelled, olfactory evidence of the presence of isopropyl alcohol was found. Inquiries quickly revealed that a solution containing 70 per cent isopropyl alcohol was used to clean the anaesthetic circuit components. Evidently, residual isopropyl alcohol in recently cleaned anaesthetic circuitry can cause factitious anaesthetic agent concentration readings.

Subsequent discussions with Puritan Bennett Limited concerning this matter revealed the following informa- tion. The problem is known to them, and is a characteristic believed to be common to all anaesthetic agent monitors which use infrared spectrophotometric methods, not just the Puritan Bennett unit. Not only will evaporated alcohol or other organic vapours affect the readings, but readings from patients with alcohol in their blood will be higher than the actual anaesthetic agent concentration.

D.J. Doyle MD PH D FRCPC

Department of Anaesthesia

Toronto General Hospital

200 Elizabeth Street

Toronto, Ontario M5G 2C4

\section{Safe vaporizers}

\section{To the Editor:}

Anaesthetic vaporizers may, inadvertently, be left "on" during an anaesthetic, with disastrous consequences for the patient.

The current Canadian Standards Association Standard applicable to vaporizers states that the control dial should be marked to indicate vapour concentration in volume per cent and the OFF position, with a lock in the OFF

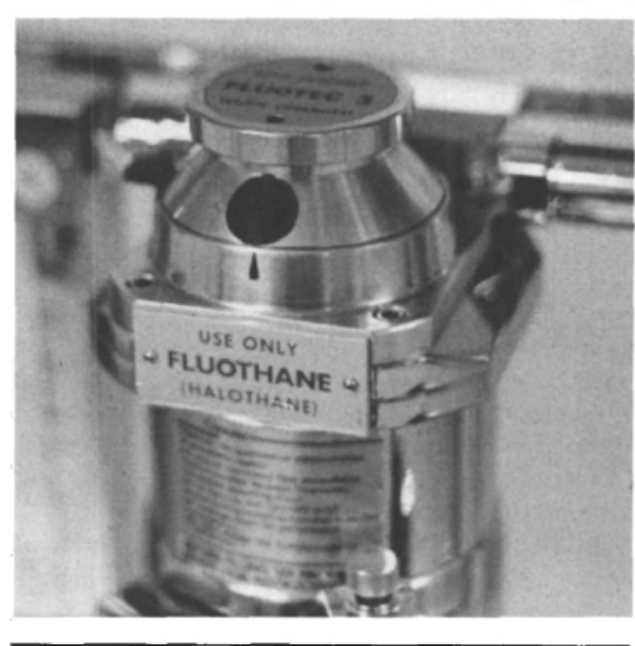

FIGURE 
position. These marks are clear, but at the usual working distance of the anaesthetist from the machine, the position of the control dial can be overlooked.

A coloured sticker placed at the OFF position on the control dial makes it obvious that the vaporizer is "off", or turned "on." In comparison with other safety devices for anaesthetic machines this is simple, cheap, and available to all anaesthetists.

Geoffrey G. Coleshill MB CH в DA

Central Interlake Clinic

Teulon, Manitoba, ROC 3B0

\section{Malfunctioning unidirectional valves of Ohmeda Series 5 and 5 A carbon dioxide absorbers}

To the Editor:

Coincidental with the introduction of a shared mass spectrometer for respiratory and anaesthesia gas analysis into the main operating room suite at the Royal Victoria Hospital, was the introduction of Ohmeda Series 5 carbon dioxide absorbers onto 15 Boyle $\mathrm{M}$ anaesthesia machines and the subsequent conversion from Mapleson D to semi-closed circuit for delivery of anaesthesia. Fresh gas flow was delivered through MDM flow meters and was approximately $2-3 \mathrm{~L} \cdot \mathrm{min}^{-1}$.

The on-line detection of inspired and expired carbon dioxide enabled us to detect the repeated malfunction of the unidirectional valves of many of these absorbers, such that wo instituted a survey of the problem during a three-week period. Fourteen incidents of malfunctioning unidirectional valves on nine units were reported involving both the inspiratory (five) and expiratory (nine), valves.

Our ability to detect this problem was entirely dependent on the niass spectrometer which was programmed to aler the anaesthetist at a $\mathrm{PE}^{\prime} \mathrm{CO}_{2}>5 \mathrm{mmHg}$. It was this programmed alert which prevented, as far as we were aware, damage to our patients, but clearly the situation was far from satisfactory. We were consequently obliged to inform the manufacturer (Ohmeda, Rexdale) and Health and Welfare Canada via their Medical Devices Problem Reporting Program of our experiences and request a solution to the problem.

Investigation of the problem by the company revealed reproducible malfunction of the valve assembly. Ohmeda determined that this was the result of the flow characteristics of the gases in the breathing circuit, in combination with the effects of surface tension from the accumulation of condensation on the valve components.
Subsequent redesign of the valve disc, disc cage and valve seat of the unidirectional valve assembly preceded field testing of the new components during which no incidence of malfunction was reported. Consequently, an urgent Medical Device Recall was issued by Ohmeda in May 1988 to all users of Series 5 and 5A Carbon Dioxide Absorbers calling attention to the problem and offering replacement of the existing valve assemblies.

We have used the redesigned valve system on these absorbers since January 1988, and are satisfied that the problem we have described has been resolved.

David $G$. Whalley MB CH B FFARCS FRCPC

Department of Anaesthesia

Royal Victoria Hospital

687 Pine Avenue West

Montréal, Québec H3A 1A1

\section{REPLY}

Ohmeda sincerely appreciates $D r$. Whalley's cooperation and assistance in discussing his experiences with us. Such communication is essential for manufacturers such as Ohmeda to respond to the ever-changing clinical requirements of the profession with innovative product evolution and development.

In this irstance, Oluneda has released an aduanced design of unidirectional valve for Carbon Dioxide Absorbers. This valving system is available to Canadian users of Ohmeda Series 5 and $5 A$ Absorbers in accordance with the following Recall Letter dated May 31, 1988.

\section{UAGENT - MEDICAL DEVICE HECALL}

Ohmeda (Medishield) carbon dioxide absorbers Series $5,5 A$ only

Several users of Series 5 abscrbers have reported occasionally monitoring higher than expected $\mathrm{CO}_{2}$ levels in the breathing circuit gases during controlled ventilation.

These reports indicated intermittent sticking in the open position of the mica disc used in the inhalation and exhalation valves

No patient injury has been reported and in each report the $\mathrm{CO}_{2}$ levels were restored to expected levels when the mica dise operation returned to normal ei ther by its own accord or by user intervention such as tapping the valve housing to free the disc from its "sticking" position.

Ohmeda research suggests this inhalation/ exhalation valve mal function is a result of the flow charasteristics of the breathing circuit gases when combined with the sur face tension effects of condensate accumulations on the ultra light mica valve diso, valve disc oage and valve seat assemblies.

As a result of this research, Obmeda has redesigned these portions of the inhalation/ exhalation valves to minimize the effects of condensate on valve operation.

To avoid the potential of higher than anticipated $\mathrm{CO}_{2}$ levels resulting from this mal function and to 